\title{
Über den Einfluss der intravenösen Zufuhr grosser Mengen Serumeiweisses auf das Eiweiss und den kolloid=osmo= - tischen Druck des Blutes in normalen sowie pathologischen Zuständen.
}

III. Versuch an anämischen Kaninchen.

\author{
Von \\ Gen Koseki \\ (小辡元) \\ (Aus der Medizinischen Klinitk von Prof. Dr. T. Kato, \\ Tohoku Reichsuniversität zu Sendai.)
}

Dass bei Anämien am Bluteiweissbild nicht allein quantitativ, sondern auch qualitativ beträchtliche Veränderungen zustande kommen, ist durch zahlreiche Untersuchungen klargestellt worden. Githens'), Morawitz's), Inagaki's), Gutzeit), Gottschalku. Nonnenbruch ${ }^{5)}$, Ozawa ${ }^{6}$, Motai ${ }^{7)}$, Henriques u. Klausen ${ }^{8)}$, Cuvelier u. Potoir ${ }^{9)}$, Yosida ${ }^{10)}$ u. a. m. haben den Nachweis erbracht, dass bei durch Aderlass erzeugter Anämie das Gesamteiw eiss des Blutes abnimmt, ferner starke Abnahme des Globulins und relative Zunahme des Albumins vorkommen, welch letzte aber recht kurze Zeit anhält, und daran anschliessend starke Zunahme des Globulins auftritt. Fis hberg ${ }^{11)}$ sah, als er durch wiederholte Entnahme von relativ kleinen Blutmengen die Anämie provozierte, die Abnahme des Serumeiweisses mit gleichzeitiger relativer Globulinzunahme auftreten. Saito ${ }^{12)}$,

1) Githens, Beitr. z. chem. Physiol. u. Path., 1904, 5, 515.

2) Morawitz, Ibid., 1906, 7, 153.

3) In a gak i, Ztschr. f. Biol, 1907, 49, 77.

4) Gutzeit, Dtseh. Arch. f. klin. Med., 1924, 143, 238.

5) Gottschalk u. Nonnenbruch, Arch. f. exp. Path. u. Pharm., 1923,96,115.

6) $\mathrm{Oz} \mathrm{a} \mathrm{wa,} \mathrm{Fukuoka} \mathrm{Ikadaigaku} \mathrm{Zassi,} \mathrm{1926,} \mathrm{19,} 591$.

7) Motai, Aichi Ikadaigaku Zassi, 1930, 37, 1158.

8) Henriques u. Klausen, Biochem. Ztschr., 1932, 254, 414.

9) Cuvelier u. Potoir, C. r. Soc. Biol, 1932, 111, 16.

10) Yosida, Kumamoto Igakkai Zassi, 1940, 16, 259.

11) Fish berg, Biochem. Ztschr., 1928, 195, 20.

12) Sa ito, Tohoku Journ. Exp. Med., 1932, 19, 445. 
Sanada, ${ }^{13)}$, und Yasuda ${ }^{14}$ an hiesiger Klinik haben bei Anämien Herabsetzungen des Serumeiweisses sowie kolloid-osmotischen Drucks (k. o. D.) festgestellt und diese darauf zurückgeführt, dass beim Aderlass aus den Eiweissdepots, wie Leber und Muskel u. a. das Albumin ins Blut übertritt, dass es aber bei wiederholten Entnahmen von grossen Blutmengen gleich darauf zum Mangel des Albumindepotes in Geweben kommt, worauf das Globulin mobilisiert wird. In der Folge hat S anad a ${ }^{13)}$ bei Anämien neben der Bestimmung des Bluteiweisses und dessen k. o. D. auch die Bestimmungen des Gewebseiweisses in Leber, Niere und Hirn durchgeführt und hier ebenfalls oben erwähnte Angaben bestätigt.

Onozaki ${ }^{15}$ hat bei durch Aderlässe erzeugter Anämie die Bluttransfusion vorgenommen und gefunden, dass die Herabsetzungen des Serumeiweisses sowie des k. o. D. durch Anämie in kurzer Zeit wiederhergestellt wurden. Es drängte sich uns die Frage auf, ob und in welcher Weise Serumeiweiss und k. o. D. dann beeinflusst würden, wenn bei Anämien grosse Dosen Serumeiweisses intravenös verabreicht worden sind. Aus dieser Überlegung wurde vorliegende Untersuchung in Angriff genommen.

Technische Massnahme der Injektion von Serumeiweiss und die Methode zur Bestimmung an gewonnenen Blutproben waren ganz dieselben, wie die in der I. Mitteilung ${ }^{\mathbf{1 6})}$ geschilderten.

Die Bestimmungen wurden einmal vor der Erzeugung der Anämie und in der Seruminjektion unmittelbar vorangehender Zeit, weiterhin 1, 3, 6 Std. später und vom darauffolgenden Tag an einmal täglich bis 7 Tage morgens nüchtern ausgeführt.

\section{Kontrollversuch.}

Es wurde Kaninchen durch wiederholte Blutentnahme hochgradige Anämie herbeigeführt und die nach Abschluss der Blutentnahme spontan erfolgende Wiederherstellung des Bluteiweisses und dessen k. o. D. wurde verfolgt. Hier erhaltene Resultate dienten als Kontrolle gegenüber dem Hauptversuch der Seruminjektion.

Als Versuchsobjekt wurden 4 gut ernährte Kaninchen benutzt. Diese Kaninchen wurden von der Ohrvene aus je 15-20 ccm Blut jeden Tag um den andern solange entnommen, bis nach ca 2 Wochen

13) S a n a d a, Tohoku Journ. Exp. Med., 1936, 29, 144.

14) Y a suda, Ibid., 1937, 31, 437.

15) On oz a k i, Ibid., 1935, $25,1$.

16) Ko s e ki, Ibid., 1941, 39, 533. 
entnommene Gesamtblutmenge 110-130 ccm erreichte und das Hämoglobin ungefähr auf die Hälfte seines Ursprungswertes reduziert war. Es wurden dann die Veränderungen des Serumeiweisses sowie des k. o. D. dem Verlauf nach beobachtet. Die Resultate sind in Tab. 1 aufgezeichnet. Als ein Beispiel hierfür ist Versuch 2 in Fig. 1 demonstriert.

Fig. 1. Prozentnelle Vepänderungen des Hämoglobins, des Serumeiweisses, des kolloid-osmotischen Drucks und des Druck pro\%ó des Blutes bei Anämie (Versuch 2).

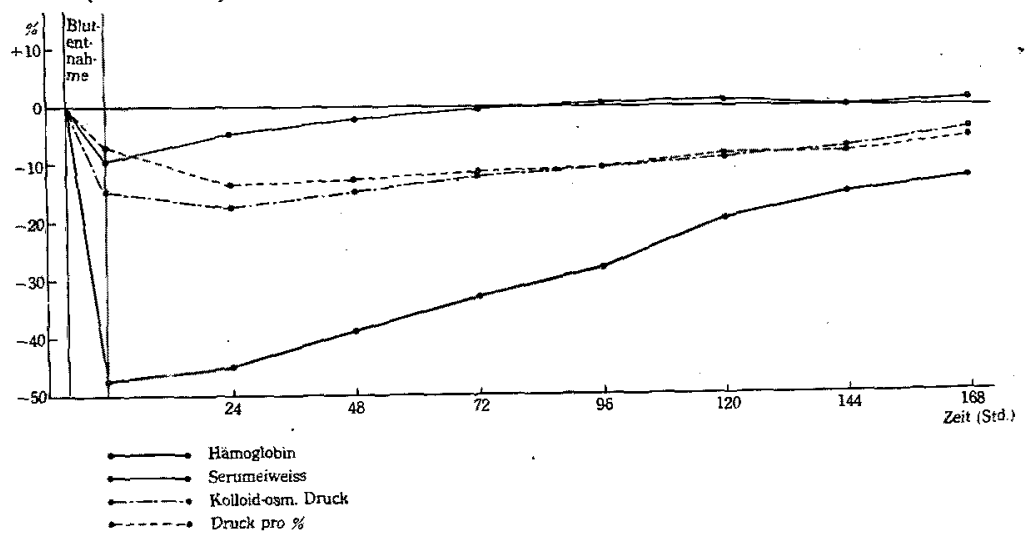

Ein Vergleich der Werte, die an Blutproben in 24-stündigem Ablauf nach letztester Blutentnahme ermittelt wurden, mit entsprechenden Werten vor Anämie ergab: Serumeiweiss nahm um 4,7-10,3\% ab, k. o. D. war auch um 11,5-15,5\% vermindert. Da die Abnahme des k. o. D. grösser als die des Serumeiweisses war, erfuhr k. o. D. für $1 \%$ Eiweiss (Druck pro \%) eine Herabsetzung um 5,4-7,2\%. Hümoglobin erfuhr eine Abnahme um 45,1-50,1\% welche weitgehend erheblicher war als die Abnahme des Eiweisses. Wenn man den Zeitpunkt, wo die erstmalige Blutprobe nach Anämie vorgenommen wurde, als zeitliches Standard den weiteren Verlauf der Veränderungen beobachtet, so ergibt sich folgendes: Serumeiweiss wurde relativ rasch wiederhergestellt, indem er nach $72 \mathrm{Std}$. annähernd das Niveau vor Anämie erreichte. K. o. D. war hingegen an Wiederherstellung stark gehindert, so dass er selbst nach einer Woche noch nicht vollkommen wiederhergestellt wurde. Da die Wiederherstellung des k. o. D., wie oben erwähnt, langsamer als die des Serumeiweisses von statten ging, verzögerte sich auch die Wiederherstellung des Drucks pro \%. Druck pro \% tendierte nämlich im Anfangsstadium eine Zeitlang eher zur 


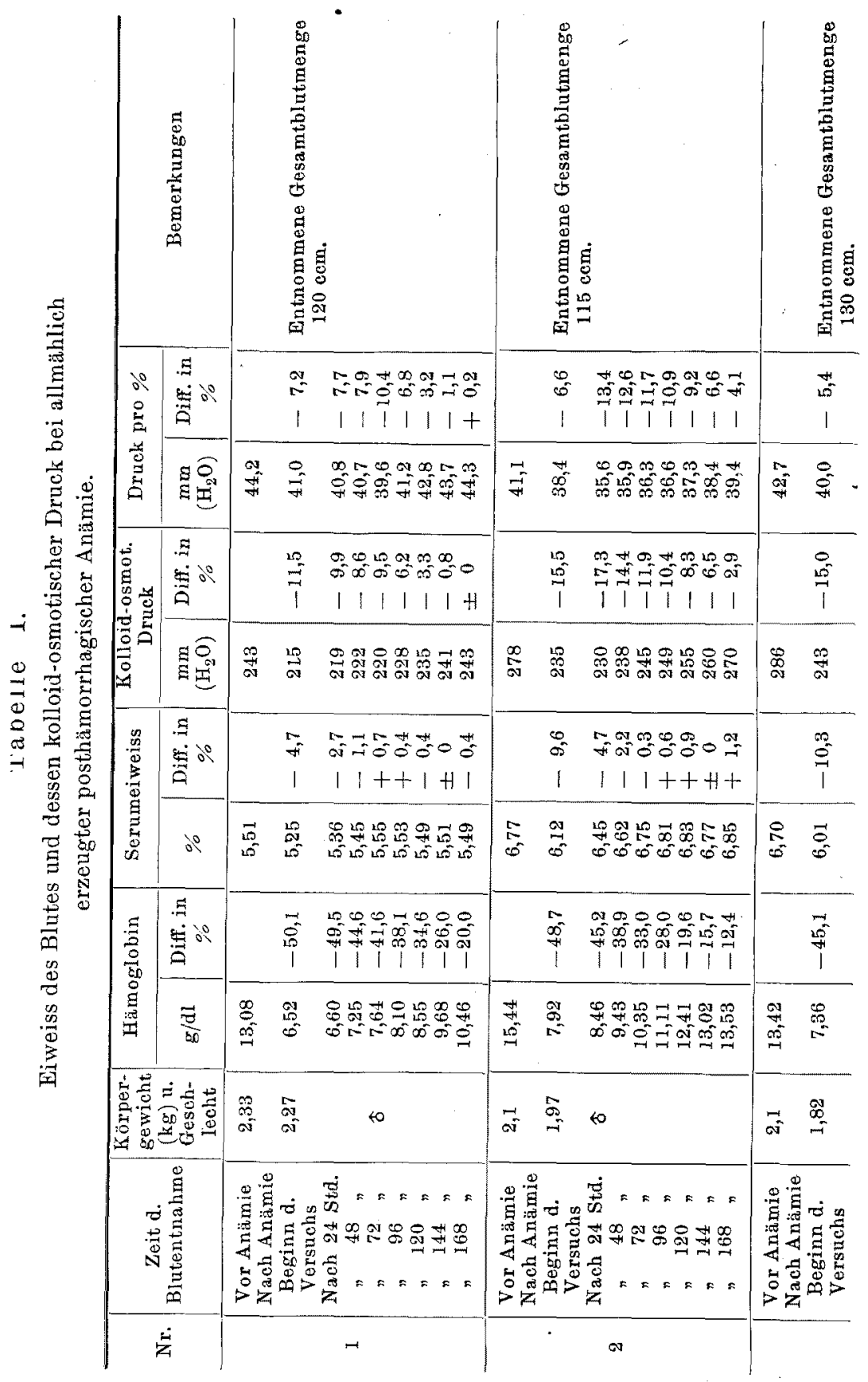




\begin{tabular}{|c|c|c|}
\hline & 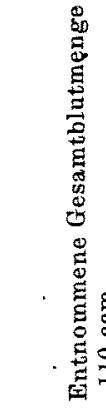 & \\
\hline 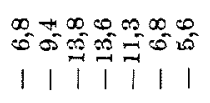 & $\begin{array}{l}0 \\
0 \\
0\end{array}$ & 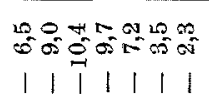 \\
\hline 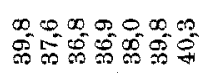 & $\vec{c}$ & 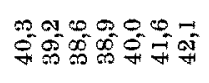 \\
\hline 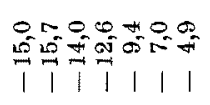 & & 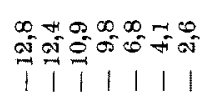 \\
\hline 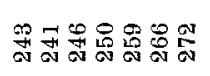 & 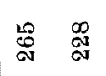 & 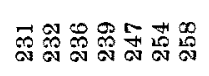 \\
\hline 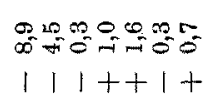 & $\begin{array}{c}\vec{x} \\
1\end{array}$ & 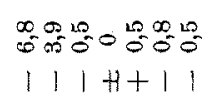 \\
\hline 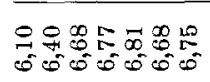 & $\underset{10}{2}: 0$ & 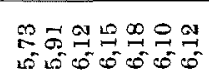 \\
\hline 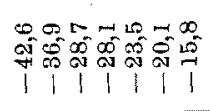 & & 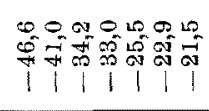 \\
\hline 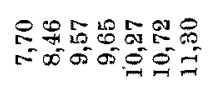 & 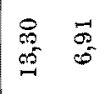 & 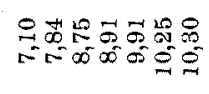 \\
\hline to & $\stackrel{0}{\circ} \underset{0}{0}$ & of \\
\hline 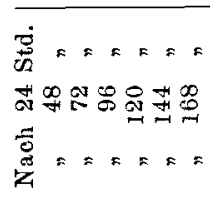 & 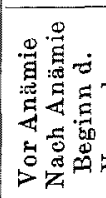 & 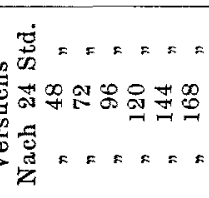 \\
\hline & & \\
\hline
\end{tabular}

Herabsetzung, indem er bei Versuch 2 nach 24 Std. bei übrigen Versuchen nach 72 std. minimale Werte aufwies, worauf er dem Anfangswert zustrebte; dennoch zeigte er ausgenommen Versuch 1, wo nach einer Woche annähernd der Wert vor Anämie erreicht wurde, bei übrigen Versuchen noch eine Abnahme von 2,3-4,1\%.

Die Unterbrechung wiederholter Blutentnahme führt zu verhältnismässig rascher Wiederherstellung des Serumeiweisses, da aber diese Wiederherstellung vorwiegend durch die Eiweissfraktion des Globulinanteils bewirkt wird, so hält k. o.D. gleichen Schritt damit nicht, sondern verzögert sich in der Wiederherstellung, wodurch Druck pro \% herabgedrückt ist. Nachher aber, nachdem das Serumeiweiss das Anfangsniveau erreicht hatte, fängt Druck pro \% an Hand in Hand mit dem Serumeiweiss anzusteigen, um nach einer Woche, wenn auch nicht vollkommen, doch dem Anfangswert ziemlich nahezukommen. Die Wiederherstellung des Hämoglobins erfolgt so langsam, dass es selbst nach einer Woche noch eine Abnahme von 12,4-21,5\% aufweist.

Nach diesem Ergebnis ist die Abnahme des Serumeiweisses gegenüber der Abnahme des Hämoglobins äusserst spärlich. Diese gelinge Abnahme liegt daran, dass das durch Blutentnahme ver- 
loren gegangene Serumeiweiss durch das aus der Leber und anderen eiweissbereitenden bzw. regulierenden Organen mobilisierte oder neugebildete Eiweiss ersetzt worden ist. Weil indessen die Eiweissorgane infolge der ziemlich grossen Eiweissquantitäten, die hierbei

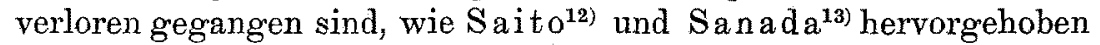
haben, keine Zeit haben, reife, kleinmolekuläre Eiweissteilchen zu mobilisieren und sich deshalb mit der Mobilisierung der grobmolekulären Eiweissteilchen des Globulinanteils behelfen, dürfte sich die Wiederherstellung des $k$. o. D. verzögern, wobei sich auch Druck pro\% dementsprechend im Anfangsstadium eher herabsetzt. Erst dann aber wenn sich das Serumeiweiss quantitativ wiederhergestellt hat, dürfte das fortan zu mobilisierende Eiweiss allmählich in kleine und feine Form übergeführt werden, was naturgemäss die Erhöhung des k. o. D. und Drucks pro \% bewirkt.

2. Versuch mit Seruminjektion.

Bei Kaninchen, die durch gleiches Verfahren wie bei dem Kontrollversuch anämisiert wurden, wurden sofort nach Entnahme der Blutproben in 24-stündigem Ablauf nach letzter Blutentnahme die Injektionen von $10 \mathrm{ccm}$ Seren pro kg Körpergewicht in üblicher Weise unternommen. An 5 Kaninchen gewonnene Resultate sind in Tab. 2.

Fig. 2. Prozentuelle Veränderungen des Hämoglobins, des Serumeiweisses, des kolloid-osmotischen Drucks und des Drucks pro \% des Blates nach Seruminjektion bei Anämie (Versuch 1).

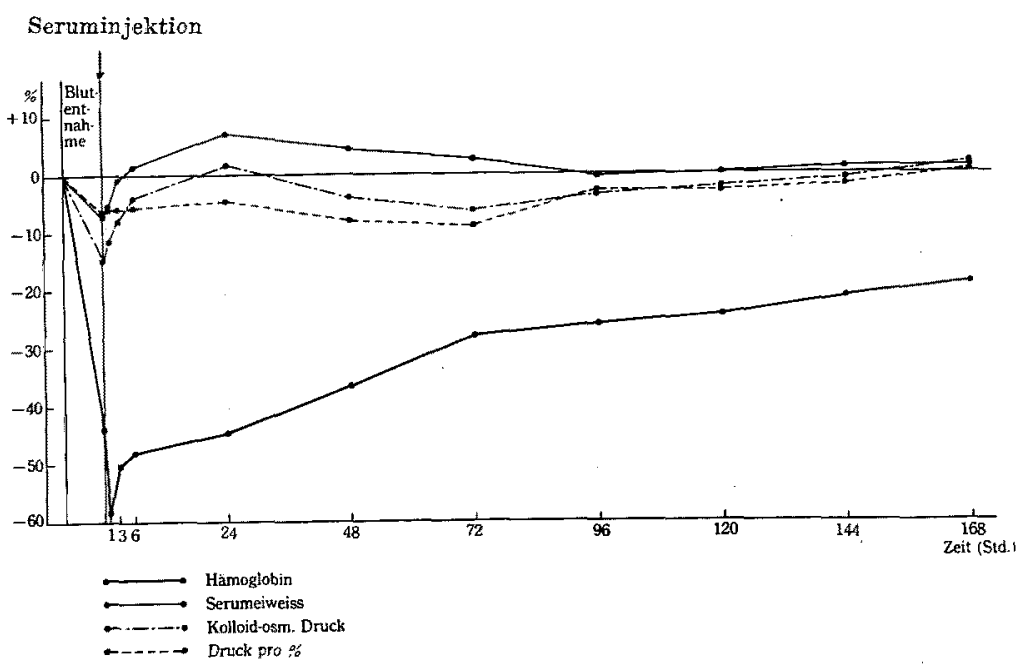


zusammengestellt. Anbei ist ein Beispiel (Versuch 1) hierfür in Fig. 2 demonstriert.

Serumeiweiss, welches durch Anämisierung eine Abnahme von 7,1-9,0\% erlitten hatte, stieg durch Injektion rasch an, d. h. war an Abnahme reduziert. Die Abnahme betrug nämlich nach 1 Std. 5,1-6,7 $\%$, nach 3 Std. nur 1,1-5,2\%. Weiterhin nach 6 Std. wurde bei Versuch 2-5 immerhin noch eine Abnahme von 0,3-1,2\% ermittelt; bemerkenswerterweise brachte das Serumeiweiss bei Versuch 1 es soweit, über das Niveau vor Anämie hinaus eine Zunahme von 1,2\% zu behalten. Nach 24 Std. trat allerdings die Maximalzunahme von 3,5-6,9\%. Seither erreichte Serumeiweiss untex allmählicher Abnahme schliesslich nach $96 \mathrm{Std}$. den annähernden Wert vor Anämie.

K. o. D., der durch Anämisierung um 14,1-15,5\% abgenommen hatte, nahm durch Seruminjektion ebenso wie Serumeiweiss zu und reduzierte sich an Abnahme. So zeigte er z. B. eine Abnahme nach 1 Std. um 10,8-13,0\%, nach 3 Std. um 6,7-6,9\%, nach 6 Std. um 3,37,1\%. Nach 24 Std. wurde das Maximum erreicht, indem. k. o. D. bei Versuch 4 noch eine Abnahme von 1,6\%, bei allen übrigen Versuchen eine Zunahme von $0-2,9 \%$ darbot. Von diesem Zeitpunkt ab schlug k. o. D. ständig bis zu $72 \mathrm{Std}$. den Weg zur Erniedrigung ein, bis er aber wieder anstieg und schliesslich nach 144 Std. annähernd auf das Niveau vor Anämie wiederhergestellt wurde. Druck pro \% erfuhr, weil die Abnahme des k. o. D. durch Anämie grösser als die des Serumeiweisses war, eine Abnahme von $6,9-8,1 \%$. Aus dem Grunde aber, dass die Reduzierung des k. o. D. und des Serumeiweisses an ihren prozentischen Abnahmen, welche durch Seruminjektion verursacht wurde, bis zu 24-stündigem Ablauf miteinander nahezu parallel ging, war Druck pro \% derart wenig an seiner prozentischen Abnahme reduziert, dass Druck pro \% noch eine Abnahme von 3,2-5,9\% beibehielt, abgesehen von Versuch 2, wo die Abnahme durch Anämie von 6,9\% auf $0,5 \%$ reduziert war, eine Erhöhung, welche immerhin als mehr oder weniger beachtenswert bezeichnet werden kann. Da die Herabsetzung des k. o. D. von 24-stündigem bis zu 72-stündigem Ablauf etwas grösser als die Abnahme des Serumeiweisses war, setzte sich Druck pro \% mehr oder weniger herab und erfuhr nach 72 Std. eine Abnahme von 5,7-10,4\%. Seither trat Druck pro \% parallel mit dem k. o. D. an das Anfangsniveau heran und kam nach 144 Std. annähernd bis zu dem präanämischen Niveau zurück.

Hämoglobin, obwohl es durch Seruminjektion an seiner Konzentration herabgesetzt war, kam nach 24 Std. dem der Injektion unmittel- 


\begin{tabular}{|c|c|c|c|}
\hline \multirow{12}{*}{ 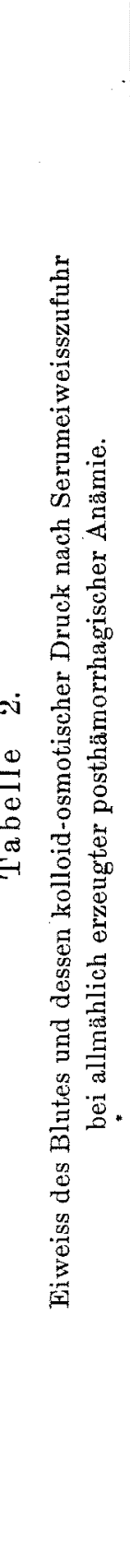 } & 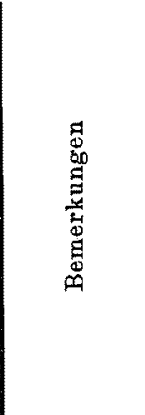 & 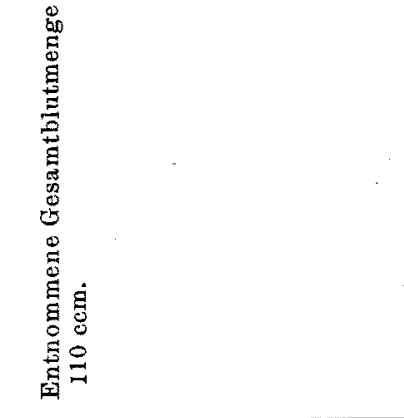 & 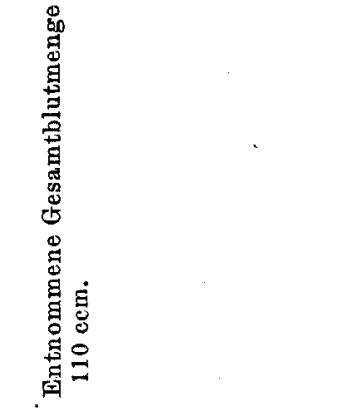 \\
\hline & 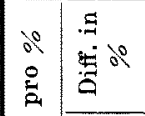 & 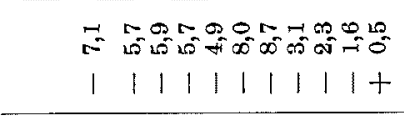 & 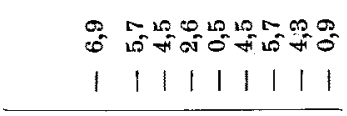 \\
\hline & 总总 & 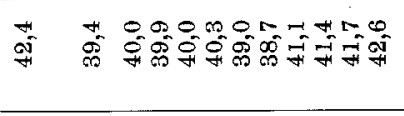 & 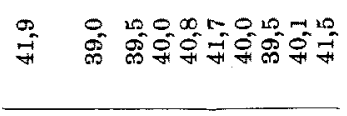 \\
\hline & 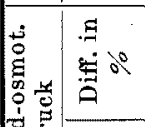 & 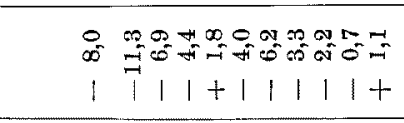 & 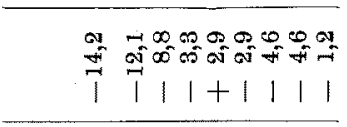 \\
\hline & है & 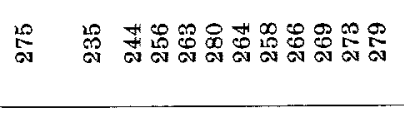 & 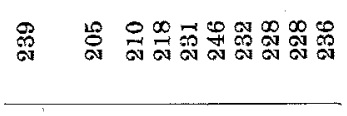 \\
\hline & 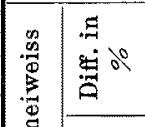 & 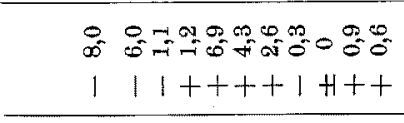 & 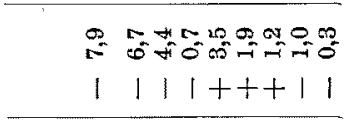 \\
\hline & 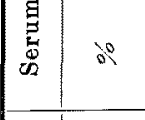 & 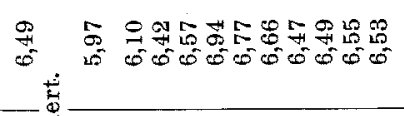 & 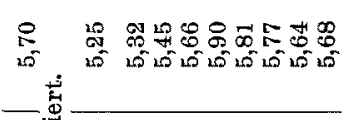 \\
\hline & 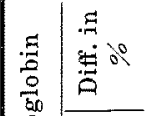 & 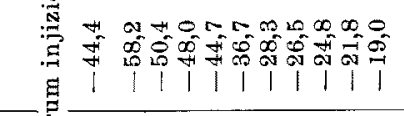 & 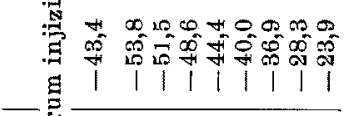 \\
\hline & 㓪 & 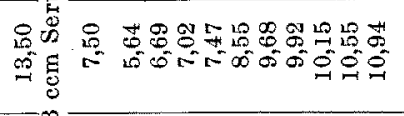 & 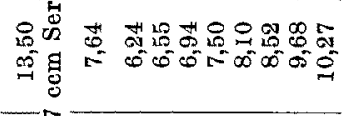 \\
\hline & 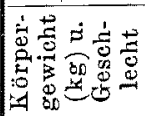 & $=0$ & 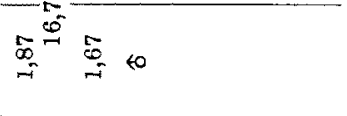 \\
\hline & 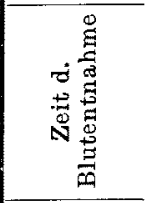 & 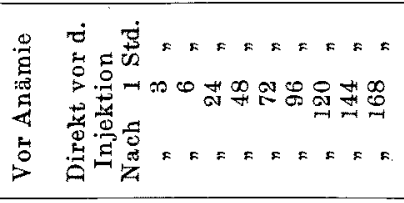 & 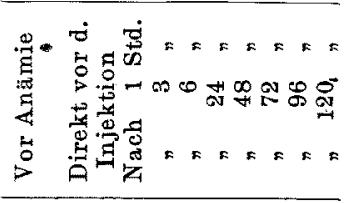 \\
\hline & $\dot{\vec{z}}$ & $\rightarrow$ & ه \\
\hline
\end{tabular}


Einfluss der Seruminjektion auf das Bluteiweiss. III.

\begin{tabular}{|c|c|c|c|c|c|c|}
\hline & 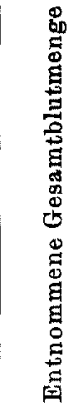 & 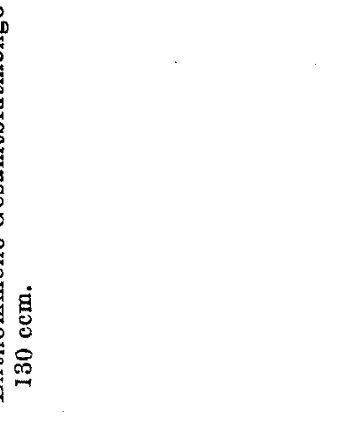 & 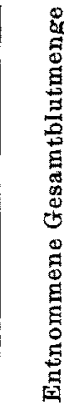 & 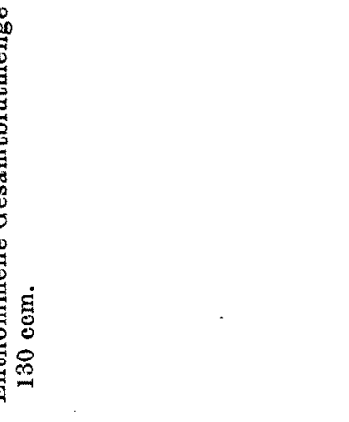 & $\begin{array}{l} \\
8 \\
0 \\
0\end{array}$ & 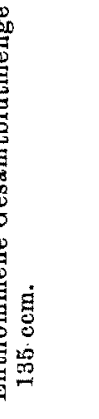 \\
\hline $\begin{array}{l}\text { ag o } \\
1+\end{array}$ & & 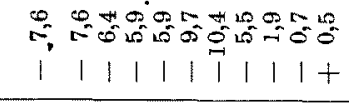 & & 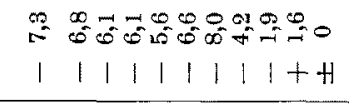 & & $\begin{array}{cc}-1 & 0 \\
\infty & 8 \\
1 & 1\end{array}$ \\
\hline 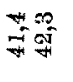 & $\overrightarrow{\widehat{T}}$ & 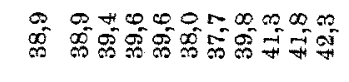 & â & 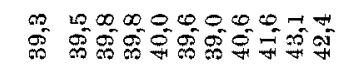 & क्ष & 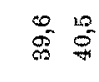 \\
\hline 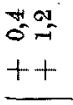 & & 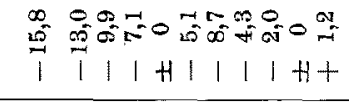 & & 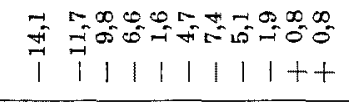 & & 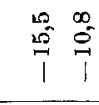 \\
\hline 융 ్ㅗㅇ & 嵒 & 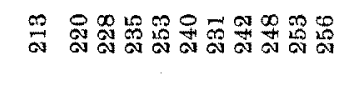 & & 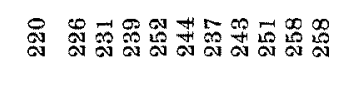 & 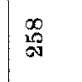 & $\stackrel{\infty}{\infty} \underset{\infty}{\infty}$ \\
\hline $\begin{array}{l}\infty=0 \\
=0 \\
++\end{array}$ & & 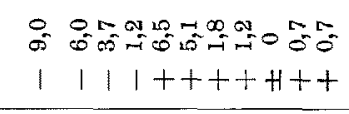 & & 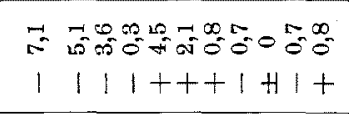 & & $\begin{array}{cc}0 \\
0 \\
1 & 0 \\
1 & 1\end{array}$ \\
\hline 空雨 & & 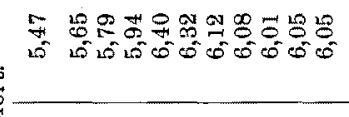 & 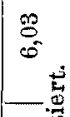 & 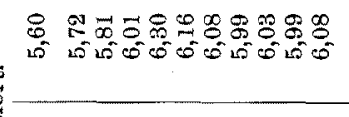 & 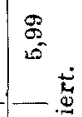 & $\begin{array}{ll}0 \\
0 \\
0 \\
0\end{array}$ \\
\hline $\begin{array}{l}8 \infty \\
0 \\
11 \\
11\end{array}$ & 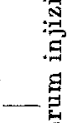 & 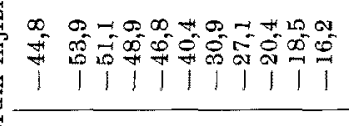 & 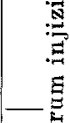 & 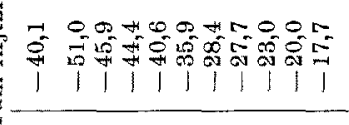 & 胥 & की \\
\hline \multirow[t]{2}{*}{ 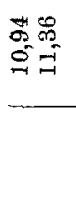 } & 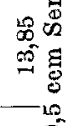 & 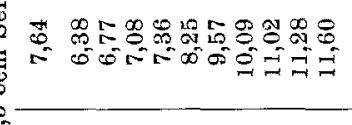 & 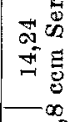 & 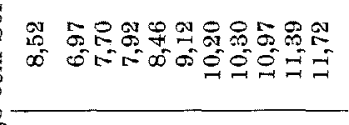 & 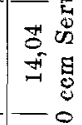 & $\begin{array}{ll}\infty & 5 \\
\infty & 0 \\
\infty & 0 \\
0 & 0\end{array}$ \\
\hline & $\stackrel{-\infty}{=}$ & $\frac{\pi}{-1}+0$ & $\stackrel{\infty}{\overbrace{-\infty}^{\infty}}$ & $\infty_{-\infty}^{\infty}$ of & $\stackrel{\infty}{\infty}$ & $\stackrel{\infty}{-1}<0$ \\
\hline \multirow[t]{2}{*}{ 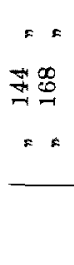 } & 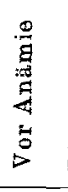 & 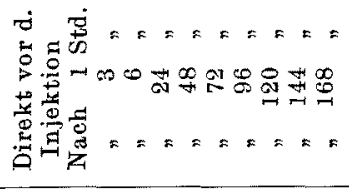 & 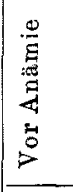 & 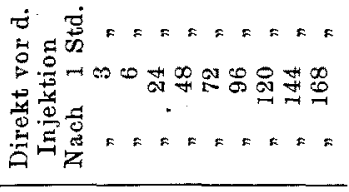 & 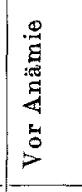 & 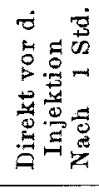 \\
\hline & & & & & & \\
\hline
\end{tabular}




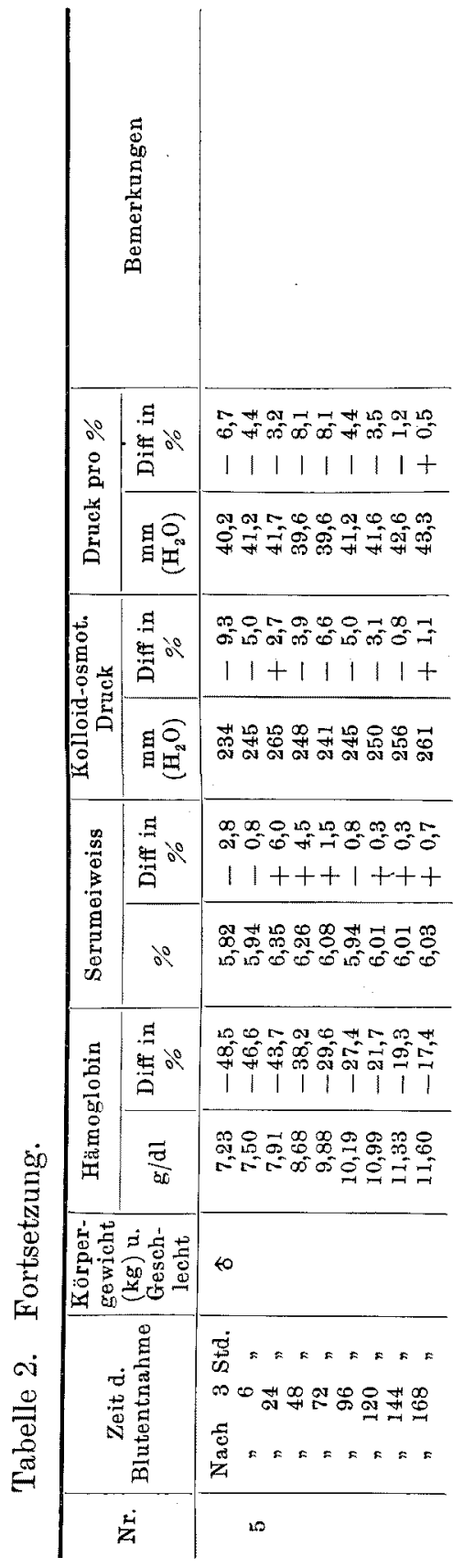

bar vorangehenden Wert nahe und stieg weiter allmählich an, um dennoch nach einer Woche noch eine Abnahme von 15,8-19,0\% beizubehalten.

Das injizierte Serum verliert anfänglich graduell an seinem Wasser und dickt sich ein, was die $\mathbf{Z u -}$ nahme der Eiweisskonzentration zux Folge hat. K. o. D. erhöht sich auch, diese Erhöhung muss jedoch, wie aus äusserst geringfügiger Zunahme des Drucks pro \% hervorgeht, vorwiegend durch Zunahme des Globulins bedingt sein. Diese Erhöhung erreicht nach 24 Std. das Maximum und schlägt dann in die Herabsetzung um, die wohl darauf beruhen dürfte, dass das konzentrierte Serumeiweiss zum Teil in die Fiweissdepots eintritt, während anderèrseits das in Gefässen zurückgebliebene Eiweiss allmählich gespalten und in den Nichteiweissstoff umgewandelt wird. Da das hierbei gespaltene Eiweiss aller Wahrscheinlichkeit nach vorwiegend zum kleinmolekulären Anteil gehört, und deshalb der relativ grossmolekuläre Anteil zurückbleibt, dürfte Druck pro \% sich herabsetzen, demgemäss mag auch k. o. D. absinken.

Da parenteral zugeführtes Serumeiweiss, wie oben erwähnt, zum Teil in die Depotorgane hineintritt und so an ihrem Eiweissbestand vermehrt, mobiliseren sich aus Depotorganen mehr osmoaktive kleinmolekuläre Eiweissteilchen als vor Serum- 
injektion; diesem Umstand dürfte wahrscheinlich das Serumeiweiss seine frühzeitige qualitative Wiederherstellung als bei der Kontrolle verdanken.

\section{Zusammenfassung.}

1. Wenn durch wiederholte Blutentziehung diejenige Anämie, bei der der. Hämoglobingehalt ungefähr bis auf die Hälfte seines ursprünglichen Wertes herabgesetzt ist, erzeugt wird, sinken im Blut Eiweiss, k. o. D. und Druck pro \% ab. Nach Unterbrechung der Blutentziehung erholt sich das Serumeiweiss quantitativ verhältnismässig rasch, wogegen k. o. D. sowie Druck pro\% sich in ihrer Wiederherstellung verzögern, derart, dass beide selbst nach einwöchigem. Ablauf einigermassen niedrigere Werte als vor Anämie aufweisen. Hämoglobin erholt sich bald nach Abschluss der Blutentnahme allmählich, zeigt jedoch nach einwöchigem Ablauf gegenüber dem präanämischen Wert ziemlich erniedrigte Werte.

2. Wenn bei der oben erwähnten Anämie intravenöse Injektion von grossen Serumdosen vorgenommen worden ist, so erholen sich Serumeiweiss sowie k. o. D. rascher als bei der Kontrolle. Bei eingehender Betrachtung stellt sich heraus: Serumeiweiss iibersteigt rasch das präanämische Niveau und gelangt nach 24 Std. zum Maximalwert. Von dieser Periode ab nimmt Serumeiweiss allmählich $a b$ und erreicht nach $72 \mathrm{Std}$. im grossen und ganzen den präanämischen Wert. K.o.D., im Anfangsstadium gleichen Schritt mit dem Serumeiweiss haltend, erhöht sich bis 24 Std. und kommt nachher zeitweise herab. Da diese Herabsetzung höhergradig als die Abnahme des Serumeiweisses ist, so sinkt Druck pro \%, der bis 24 Std. fast unverändert geblieben ist, nachher eine Zeitlang ab. In der Folgezeit aber erhöhen sich k. o. D. und Druck pro \% Hand in Hand und gelangen zum präanämischen Wert. Die Wiederherstellung der beiden auf das präanämiche Niveau vollzieht sich später als die quantitative Erholung des Serumeiweisses. Die Wiederherstellung des Hämoglobins geschieht nicht so unterschiedlich von der Kontrolle.

Herrn Prof. Nakazawa spreche ich für seine freundliche Leitung bei vorliegender Arbeit meinen herzlichen Dank aus. 